\title{
USO DE POLÍMEROS NATURAIS E MODIFICADOS COMO AGENTE AGLOMERANTE NA PELOTIZAÇÃO DE CONCENTRADO DE MINÉRIO DE FERRO*
}

\author{
Lucas Beirão do Valle ${ }^{1}$ \\ Cláudio Batista Viera² \\ Denilson Rodrigues de Araújo ${ }^{3}$ \\ Thiago Marchezi Doellinger ${ }^{4}$ \\ Anderson Lucio de Souza ${ }^{5}$
}

\section{Resumo}

Este artigo visa estudar polímeros como quitosana, amido entre outros com elevada disponibilidade como agentes aglomerantes no processo de pelotização de minério de ferro e discutir sobre o impacto da sua utilização. Os polímeros foram testados preliminarmente no teste de bancada (teste em pneu), para medir qualitativamente a tendência de aglomeração. Os polímeros com melhores resultados foram testados em escala piloto, que simula o processo industrial. Nos testes preliminares, o amido gelificado apresentou os melhores resultados de resistência física de pelotas verdes e secas. Entretanto, no teste piloto o amido não foi capaz de controlar a umidade superficial. Os outros polímeros não foram considerados promissores, principalmente devido ao controle da umidade superficial.

Palavras-chave: Pelotização; Aglomerante orgânico; Teste em pneu; Amido.

\section{USE OF NATURAL AND MODIFIED POLYMERS AS ORGANIC BINDERS IN PELLETIZING OF IRON ORE CONCENTRATE}

\section{Abstract}

This paper aims to evaluate some polymers (for example Chitosane, Starch and others) with high availability as binder in pelletizing process and discuss about binder mechanism. The polymers were tested preliminarily in wheel test to evaluate its tendency to aglomeration. The polymers with best tendency of pelletizing were evaluated in pilot test that simulates the industrial process. In preliminarily test, the gelled starch had the best physics resistance and pelletizing results, but in pilot it hadn't controlled the superficial moisture. The others polymers haven't had good results, mainly in superficial moisture control as well.

Keywords: Pelletizing; Organic binder; Wheel test; Starch.

1 Eng $^{\circ}$, estudante de mestrado Erasmus Mundus Emerald, Universidade de Liège/Universidade de Lorraine/Universidade Técnica de Lulea, Nancy, França.

2 Dr. Sc., Prof. da REDEMAT (UFOP/UEMG/CETEC) e do Departamento de Eng. Metalúrgica e de Materiais da Escola de Minas da UFOP, Ouro Preto, Brasil.

3 Dr. Sc., Prof. do Departamento de Eng. Metalúrgica e de Materiais da Escola de Minas da UFOP e Gerente da Samarco Mineração, Belo Horizonte, Brasil.

4 Eng $^{\circ}$, Chefe de Departamento de Engenharia de Processo, Samarco Mineração, Ponta Ubu, Brasil

5 Eng ${ }^{\circ}$, Engenheiro de Processo da Gerência de Engenharia de Processo, Samarco Mineração, Ponta Ubu, Brasil

\footnotetext{
* Contribuição técnica ao $44^{\circ}$ Seminário de Redução de Minério de Ferro e Matérias-primas, 15은 Simpósio Brasileiro de Minério de Ferro e 2o Simpósio Brasileiro de Aglomeração de Minério de Ferro, 15 a 18 de setembro de 2014, Belo Horizonte, MG, Brasil.
} 


\section{INTRODUÇÃO}

Os aglomerantes são utilizados na pelotização de minério de ferro para melhorar as propriedades das pelotas cruas, secas e queimadas e para garantir valores satisfatórios de resistência mecânica para o transporte subsequente e processos piro metalúrgicos [1]. A bentonita é o aglomerante mais utilizado, mas ela possui relativamente alta concentração de sílica na sua estrutura cristalina [2]. Assim, o uso da bentonita na aglomeração a frio pode ser associado à elevada contaminação de sílica na pelota queimada, com consequente decréscimo no teor de ferro e aumento no custo de operação dos reatores de redução [3].

Os aglomerantes orgânicos são materiais substitutos para a bentonita e estes já foram estudados e discutidos em diversos trabalhos [1-6]. O principal objetivo associado ao uso do aglomerante orgânico é garantir um alto teor de ferro e baixa contaminação de sílica na pelota queimada. Os aglomerantes orgânicos são promissores nestes quesitos, pois eles são degradados durante o tratamento térmico, podendo ser eliminados.

Atualmente, os aglomerantes orgânicos são comumente utilizados nos processos de pelotização industriais, especialmente devido à demanda de pelotas com baixo teor de sílica principalmente pelos reatores de redução direta [4].

Neste contexto, este artigo avalia a eficiência de diversos polímeros naturais e modificados (amido, quitosana, goma guar, subproduto da produção de açúcar e um polímero experimental), sendo pré requisito a manutenção das propriedades físicas das pelotas cruas secas e queimadas. A escolha dos polímeros e da dosagem foi feita basicamente após estudos bibliográficos e avaliação dos preços dos materiais. Outras concentrações podem vir a ser assunto de um próximo trabalho.

Os polímeros foram testados primeiramente em testes de bancada (teste em pneu) para averiguar as propriedades de aglomeração, principalmente controle da umidade superficial, capacidade de formação de pelotas e ensaios físicos nas pelotas cruas verdes e secas. Os polímeros com os melhores resultados preliminares de aglomeração foram testados em escala piloto que simula o processo industrial. Nos testes preliminares, as pelotas produzidas com o amido gelificado apresentaram resultados satisfatórios de resistência física, mas no teste piloto ele não foi capaz de controlar a umidade superficial das pelotas. Os outros polímeros não obtiveram bons resultados nos testes preliminares principalmente devido a ineficácia no controle da umidade superficial.

\section{MATERIAIS E MÉTODOS}

O minério itabirítico utilizado foi oriundo da planta de pelotização da Samarco. Antes do processo de mistura de reagentes. O pellet feed apresenta no mínimo de $65,5 \%$ de $\mathrm{Fe}$ e máximo de $2 \%$ de Si com umidade de 10\%. Outros insumos adicionados posteriormente no concentrado itabíritico foram calcário e carvão, que são rotineiramente utilizados no processo industrial. Todos os resultados discutidos neste artigo são altamente influenciados pela tipologia do minério, aditivos e umidade. Estudos posteriores com diferentes tipos de minério de ferro e parâmetros podem obter resultados diferentes.

A escolha dos aglomerantes orgânicos e dosagem foram baseadas na viabilidade econômica. Todos os testes padrões utilizaram um aglomerante orgânico aprovado para o processo industrial da Samarco.

\footnotetext{
* Contribuição técnica ao 44 Seminário de Redução de Minério de Ferro e Matérias-primas,

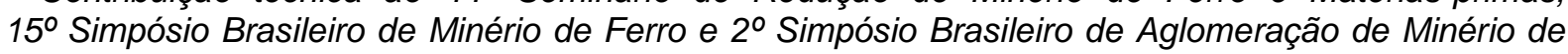
Ferro, 15 a 18 de setembro de 2014, Belo Horizonte, MG, Brasil.
} 
Os polímeros estudados foram:

- Polímero subproduto do açúcar, utilizado na indústria de refratários e possui elevado poder de aglomeração.

- Polímero experimental, indicado por especialistas da área, mas informações posteriores não foram encontradas.

- Quitosana: é o segundo polímero natural mais disponível e vem das conchas de crustáceos. Atualmente ele é utilizado no tratamento de efluentes e como material aglomerante, mas a sua melhor solubilização ocorre em pH ácido [7].

- Goma guar: foi considerado como um polímero promissor devido à capacidade de absorver umidade. Este polímero é obtido de plantas leguminosas nos EUA e Índia e sua melhor utilização é próximo à origem devido a possíveis complicações e custos de transporte [8].

- Amido: este pode ser obtido de vegetais, tais como o milho e o trigo. Sua eficiência depende da sua origem [5]. Neste estudo foi utilizado um amido modificado solúvel em água. Ele foi testado na forma gelificada e seca.

Todos os testes foram conduzidos no laboratório da Samarco Mineração em Ponta Ubu com o apoio e monitoramento da equipe altamente qualificada da empresa.

$O$ primeiro teste dos polímeros foi feito no teste em escala de bancada (teste em pneu). Este teste visa simular o rolamento de pequenas partículas de minério e grãos de pelotas sob um filme de água de maneira similar que ocorre na disco de pelotamento industrial. O teste necessita de cerca de cinco quilogramas de minério e aditivos (carvão, cal, aglomerante e água), com teores similares à alimentação industrial. A mistura é alimentada em um pneu de 39 centímetros de diâmetro com rotação e inclinação constantes e objetiva-se obter pelotas entre oito e dezesseis milímetros, que devem ser submetidas a testes de caracterização química e mecânica. O teste em pneu visa descrever a tendência de possíveis resultados em escala piloto, sendo que mesmo utilizando testes quantitativos (teste de quedas, CPCU e CPCS), os resultados representam qualitativamente o processo de pelotamento.

As pelotas obtidas no teste de pneu foram submetidas aos seguintes testes: umidade, teste de quedas e compressão de pelotas verdes e secas. Todos os testes seguiram os procedimentos internos aplicados pela Samarco. A sequência de ensaios está descrito na figura 1.

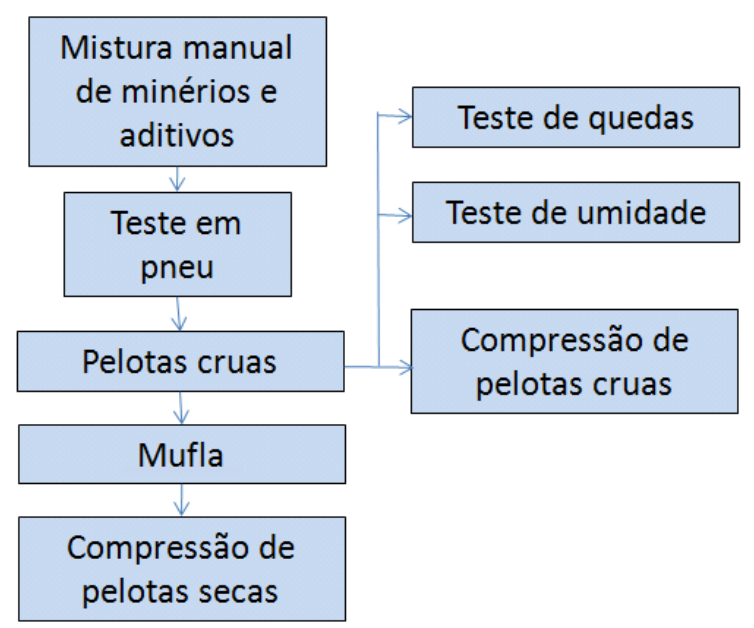

Figura 1: Metodologia do teste de bancada

* Contribuição técnica ao 44 Seminário de Redução de Minério de Ferro e Matérias-primas, 15o Simpósio Brasileiro de Minério de Ferro e 2o Simpósio Brasileiro de Aglomeração de Minério de Ferro, 15 a 18 de setembro de 2014, Belo Horizonte, MG, Brasil. 
Nestes testes foram avaliados todos os polímeros discutidos posteriormente em diferentes concentrações. A tabela 2 apresenta a nomenclatura que será utilizada neste artigo para os testes assim como o polímero e sua dosagem. A dosagem foi calculada em porcentagem em massa seca do pellet feed. Todos os testes foram seguidos de um padrão utilizando um aglomerante aprovado para o processo industrial (ou a bentonita ou o aglomerante orgânico padrão).

Tabela 2: Nomenclatura dos testes

\begin{tabular}{c|c|c}
\hline Teste em pneu & Aglomerante & Dosagem \\
\hline Test A & Subproduto do açucar & $0,400 \%$ \\
\hline Test B & Subproduto do açucar & $0,200 \%$ \\
\hline Standard 1 & Bentonita & Padrão \\
\hline Test C & Quitosana & $0,035 \%$ \\
\hline Standard 2 & Aglomerante orgânico padrão & Padrão \\
\hline Test D & Goma Guar & $0,035 \%$ \\
\hline Standard 3 & Aglomerante orgânico padrão & Padrão \\
\hline Test E & Polímero experiental & $0,035 \%$ \\
\hline Test F & Polímero experiental & $0,350 \%$ \\
\hline Standard 4 & Aglomerante orgânico padrão & Padrão \\
\hline Test G & Amido gelificado com NaOH & $0,035 \%$ \\
\hline Test H & Amido gelificado à quente & $0,035 \%$ \\
\hline Standard 5 & Aglomerante orgânico padrão & Padrão \\
\hline Test I & Amido em pó seco & $0,035 \%$ \\
\hline Standard 6 & Aglomerante orgânico padrão & Padrão \\
\hline
\end{tabular}

Assim, após a comparação dos resultados de aglomeração e de resistência das pelotas cruas e secas, os aglomerantes mais promissores foram testados em escala piloto que representa com mais fidedignidade o processo industrial.

A planta piloto apresenta as seguintes etapas: rolo de prensa, mistura, disco de pelotização e forno piloto de grelha fixa. $O$ aglomerante testado foi o amido gelificado com soda caustica. A figura 2 mostra as etapas aplicadas durante o teste piloto.

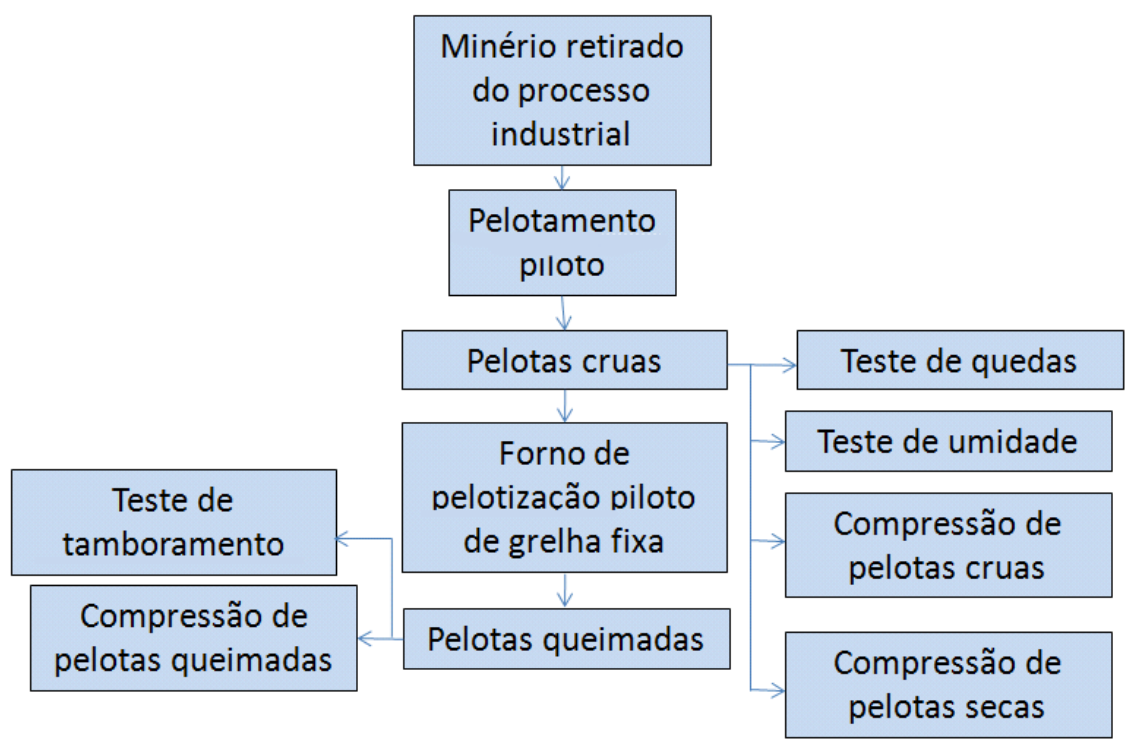

Figura 2: Metodologia do teste piloto

* Contribuição técnica ao $44^{\circ}$ Seminário de Redução de Minério de Ferro e Matérias-primas, 15o Simpósio Brasileiro de Minério de Ferro e 2o Simpósio Brasileiro de Aglomeração de Minério de Ferro, 15 a 18 de setembro de 2014, Belo Horizonte, MG, Brasil. 


\section{RESULTADOS E DISCUSSÕES}

\subsection{Testes em Escala de Bancada - Teste em Pneu}

As pelotas obtidas no teste em pneu estão mostradas na figura 3 a seguir com a nomenclatura utilizada neste trabalho.

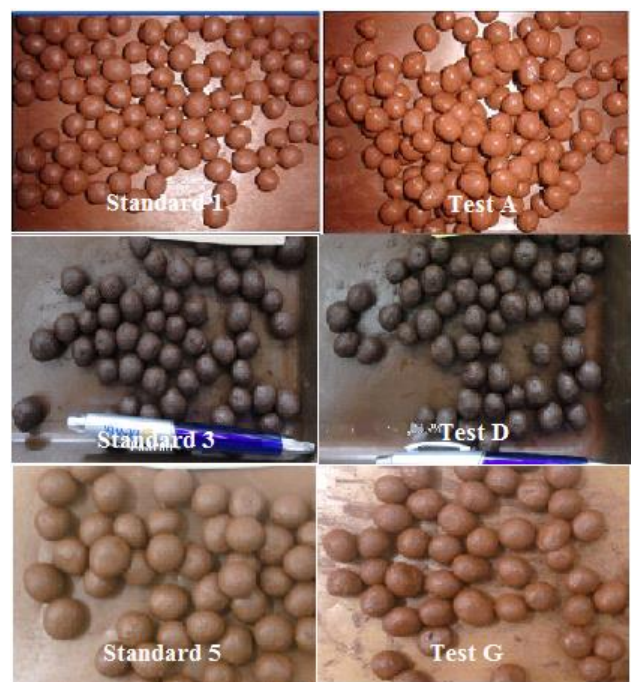

Figura 3: Fotos representativas das pelotas obtidas no teste em pneu

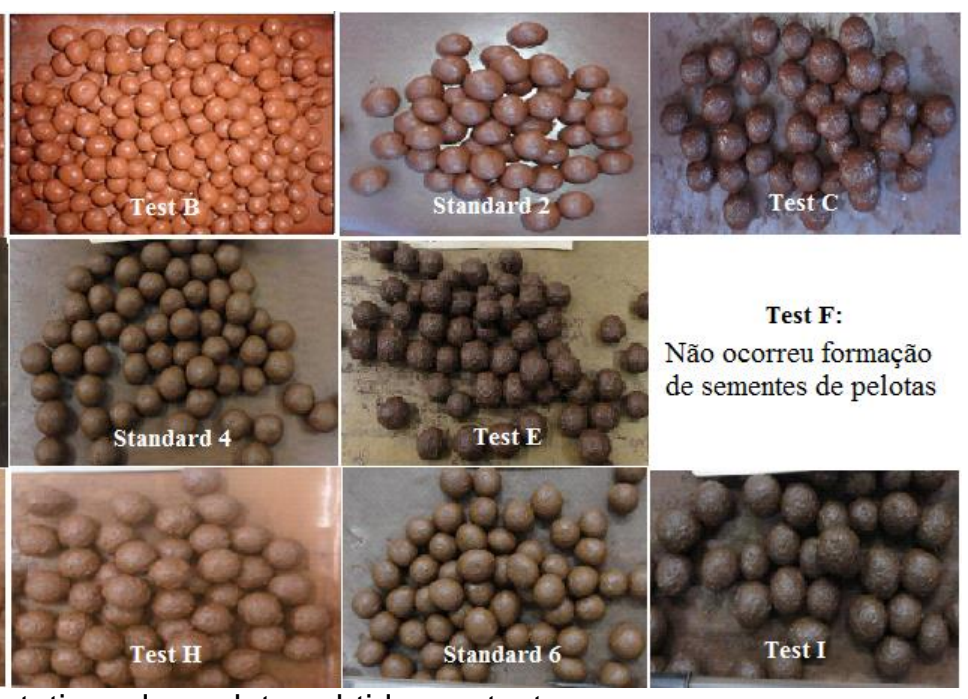

Test F:

Não ocorreu formação de sementes de pelotas

Um parâmetro importante a ser medido é a umidade superficial que é medida pela análise crítica do brilho superficial da pelota. Uma maior umidade superficial submete maior brilho à pelota.

A presença desta alta umidade superficial poderá impactar na presença de finos aderidos à superfície da pelota crua ou mesmo no aumento do fenômeno de colagem das pelotas (formação de cachos), impactando negativamente na permeabilidade do fluxo gasoso durante a etapa de queima no forno de grelha móvel. Logo, esta elevada umidade superficial deve ser evitada.

Como pode ser visto na figura 3 , quase todos os polímeros não apresentaram resultados promissores de controle de umidade superficial comparado com os padrões. Isto é, as pelotas produzidas com os polímeros apresentaram um brilho superficial significantemente maior do que o padrão, e portanto inadequado. $O$ test $D$ (que utilizou a Goma Guar) foi o único que apresentou resultados promissores comparado com o padrão. Essas conclusões foram baseadas no tipo de concentrado usado no teste e nas dosagens praticadas.

Nesta primeira análise, o polímero subproduto do açúcar foi descartado. $\mathrm{O}$ test $\mathrm{F}$ que utilizou $0,350 \%$ do polímero experimental apresentou dificuldades na taxa de crescimento das pelotas (algumas poucas pelotas cresceram demais enquanto a grande maioria permaneceu como sementes).

Os resultados dos testes resistência física das pelotas obtidas com os diversos polímeros estão apresentados na figura 4 (a), (b), (c), (d) e (e).

\footnotetext{
* Contribuição técnica ao 44 Seminário de Redução de Minério de Ferro e Matérias-primas,

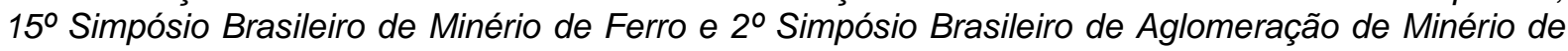
Ferro, 15 a 18 de setembro de 2014, Belo Horizonte, MG, Brasil.
} 


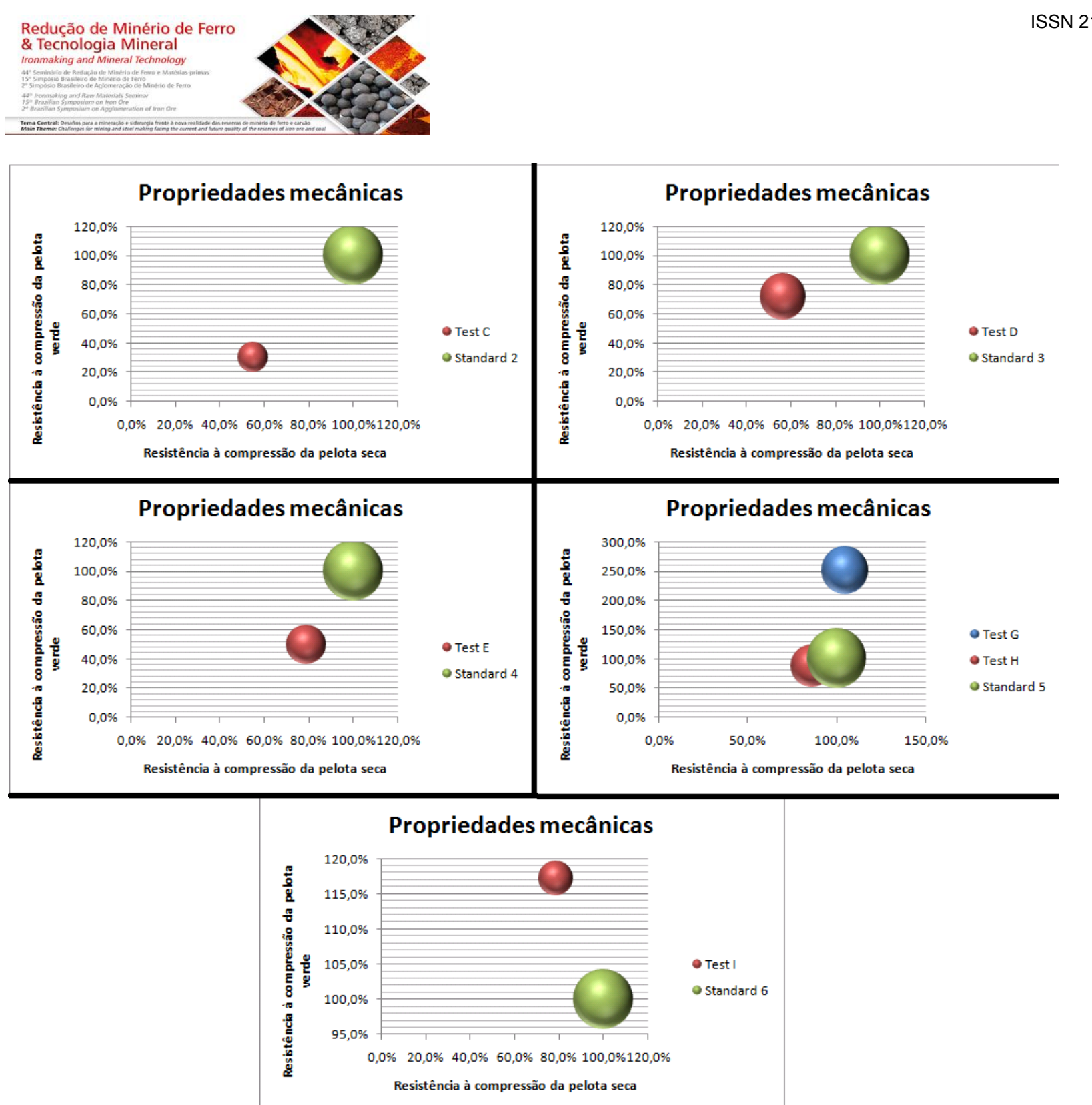

Figura 4: Resultados de resistência mecânica das pelotas obtidas em pneu

Nestas figuras estão representadas as comparações dos resultados de resistência de pelotas verdes e secas, sendo que o diâmetro do círculo é proporcional ao resultado no teste de quedas. Todos os resultados são apresentados em porcentagem relativa em comparação com os padrões (que foram plotados $100 \%$ ). Esta mesma metodologia será utilizada durante toda a discussão de dados deste trabalho.

A partir destes gráficos pode-se perceber que a quitosana (test $C$ ), goma guar (test D) e o polímero experimental ocasionaram uma perda significativa em todos os testes de resistência mecânica. Assim, eles não foram considerados promissores para este artigo nas condições estudadas. É importante salientar os resultados satisfatórios da goma guar no controle da umidade e na taxa de crescimento, mas na concentração estudada, a goma guar influenciou negativamente os resultados de resistência que não foram suficientes para suportar as condições industriais.

Os três testes utilizando o amido apresentaram resultados positivos de resistência mecânica. $\mathrm{O}$ pior foi no test $\mathrm{H}$, com o amido gelificado a quente. Este foi descartado. $\mathrm{O}$ amido seco em pó apresentou uma pequena queda na resistência à compressão da pelota verde, que pode ser associada à baixa viscosidade da solução formada com água (devido à utilização de um amido solúvel). $\mathrm{O}$ amido gelificado com $\mathrm{NaOH}$ (test $\mathrm{G}$ ) apresentou um aumento nos resultados de compressão da pelota verde e da

* Contribuição técnica ao 44 Seminário de Redução de Minério de Ferro e Matérias-primas, 15o Simpósio Brasileiro de Minério de Ferro e 2o Simpósio Brasileiro de Aglomeração de Minério de Ferro, 15 a 18 de setembro de 2014, Belo Horizonte, MG, Brasil. 
pelota seca e um pequeno decréscimo no teste de quedas, mas que foi considerado satisfatório. Preliminarmente, o test $\mathrm{G}$ não apresentou resultado satisfatório de controle da umidade superficial, mas os resultados de resistência o tornaram promissor.

\subsection{Teste Piloto}

Assim, o único aglomerante testado em escala piloto foi o amido gelificado com $\mathrm{NaOH}$. As pelotas obtidas e os resultados de resistência mecânica estão apresentados nas figuras 5 e 6 .



Figura 5: Pelotas obtidas no teste piloto
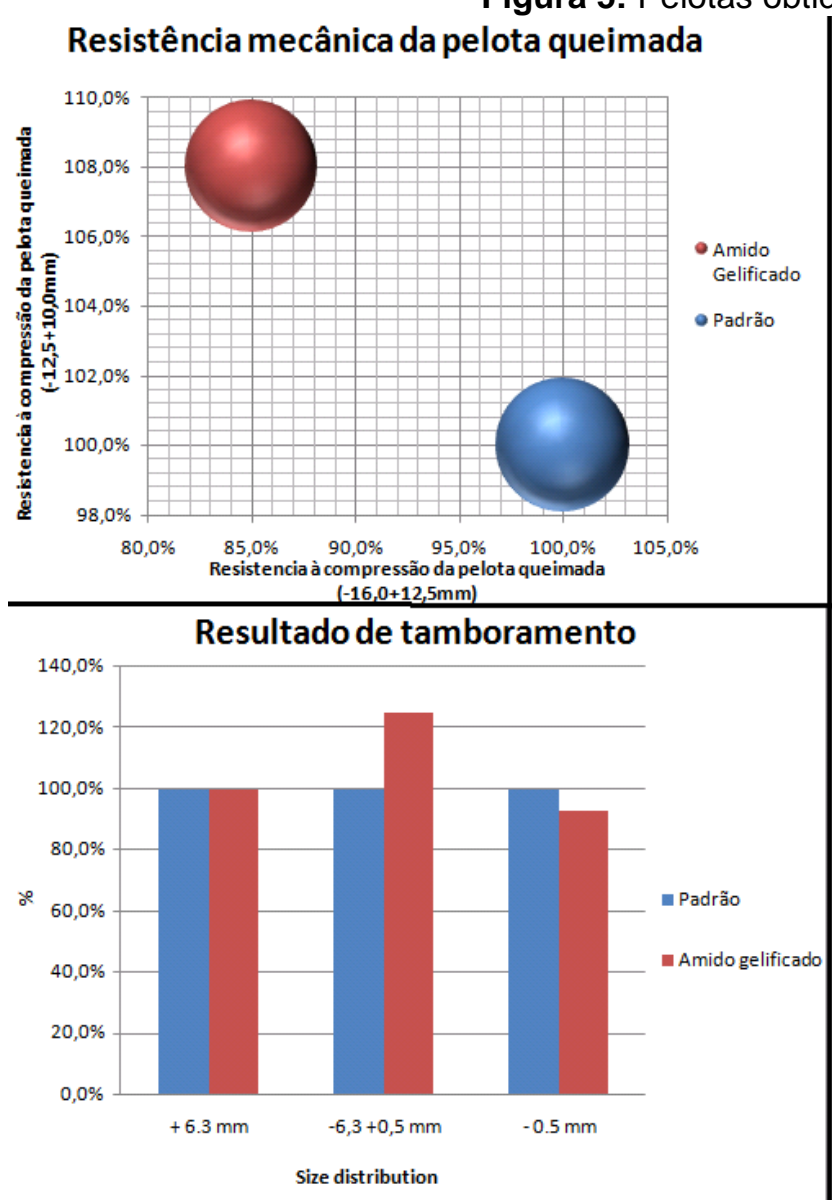

Propriedades mecânicas das pelotas verdes e secas

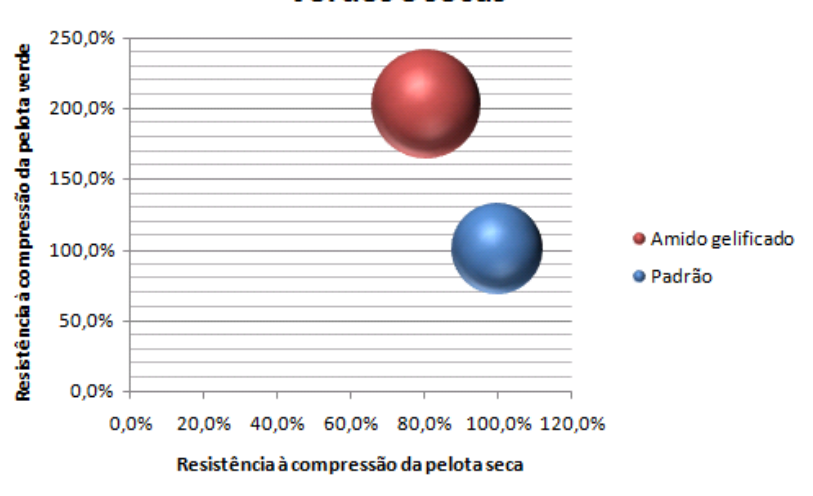

Distribuiçãogranulométrica das pelotas queimadas

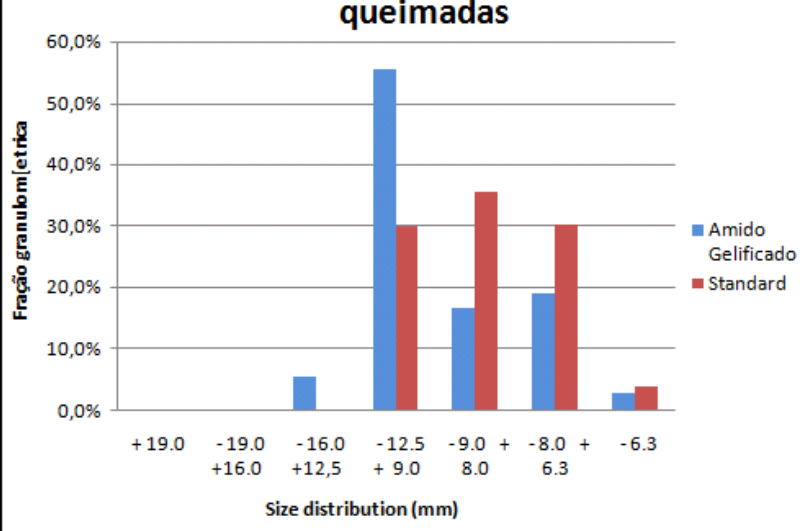

Figura 6: Resultado de resistência mecânica, química e distribuição granulométrica para as pelotas produzidas na planta piloto

Ao observar as pelotas cruas formadas na figura 5, é possível ver a formação de agregados (cachos) de pelotas no teste com amido gelificado. Com discutido anteriormente, isto não é bom para o processo seguinte de tratamento térmico das

* Contribuição técnica ao $44^{\circ}$ Seminário de Redução de Minério de Ferro e Matérias-primas, 15ㅇ Simpósio Brasileiro de Minério de Ferro e 2o Simpósio Brasileiro de Aglomeração de Minério de Ferro, 15 a 18 de setembro de 2014, Belo Horizonte, MG, Brasil. 
pelotas. Foi comprovada então a ineficácia do amido no controle da umidade superficial, o que pode ser associado à baixa capacidade de absorção de água deste amido.

O gráfico 6 (a) representa a resistência à compressão em duas faixas granulométricas, sendo o tamanho da bolha a porcentagem de sílica. $O$ teste com amido gelificado apresentou $2 \%$ de redução de sílica, mas este erro pode estar associado ao erro de medição. O gráfico 6 (b) mostra o resultado de tamboramento, enquanto que o gráfico 6 (c) representa a resistência à compressão das pelotas cruas e secas, sendo o tamanho da bolha o resultado do teste de quedas. $\mathrm{O}$ gráfico 6 (d) traz a distribuição granulométrica.

Como mostrado na distribuição granulométrica das pelotas queimadas no gráfico 6 (d), o amido gelificado apresentou a maioria das pelotas entre 12,5 e 9,0 milímetros. Isto significa que o amido controlou a taxa de crescimento das pelotas (o que é um resultado positivo) e a granulometria maior pode ser obtida variando parâmetros operacionais industriais de pelotamento, como por exemplo a taxa de alimentação e rotação do disco.

Como mostrado no teste de resistência mecânica na figura 6 (a), (b) e (c), os resultados foram satisfatórios ou melhores do que o padrão. Um porém, foi o resultado de tamboramento que mostrou uma maior tendência na geração de finos $(-6,3+0,5 \mathrm{~mm})$ durante o manuseio das pelotas.

O principal problema do uso do amido gelificado está principalmente associado ao controle e absorção de umidade. Portanto, este polímero pode ser indicado para 0 uso em um pellet feed com baixa umidade ou associado a outro aglomerante com alto poder de absorção de água (como é o caso da bentonita).

\section{CONCLUSÕES}

Nas condições estudadas neste artigo, com concentrado proveniente de um minério itabirítico das minas da Samarco, em geral, os polímeros testados não apresentaram capacidade de controle de umidade superficial e apresentariam problemas em controlar a taxa de crescimento em caso de umidade elevada do pellet feed. Outras dosagens devem ser exploradas para isso.

A goma guar foi o único capaz de controlar este fator, mas apresentou um decréscimo significativo de resistência mecânica de pelotas verdes e secas.

O amido foi o único polímero que garantiu elevada resistência para as pelotas cruas e, por isso, ele foi considerado como promissor e testado em escala piloto.

Em escala piloto, o amido novamente não controlou a umidade superficial com formação de agregados de pelotas cruas. Novamente, os resultados de resistência mecânica foram satisfatórios comparados com o padrão. Sendo assim, os resultados obtidos não foram considerados satisfatórios devido à limitação do controle da umidade superficial. Estudos exploratórios variando a dosagem dos polímeros entre outros parâmetros podem resultar em resultados satisfatórios, podendo ser assunto de um trabalho posterior.

\section{Agradecimentos}

Agradecimento à equipe da Samarco Mineração, principalmente à equipe da planta piloto pelo apoio técnico e pessoal de elevadíssima qualidade durante todos os testes.

\footnotetext{
* Contribuição técnica ao $44^{\circ}$ Seminário de Redução de Minério de Ferro e Matérias-primas, 15ํ Simpósio Brasileiro de Minério de Ferro e $2^{\circ}$ Simpósio Brasileiro de Aglomeração de Minério de Ferro, 15 a 18 de setembro de 2014, Belo Horizonte, MG, Brasil.
} 


\section{REFERÊNCIAS}

$1 \quad$ Meyer K. Pelletizing of Iron Ores. Berlin: Springer-Verlag, 1980.

2 Qiu G. Functions and molecular structure of organic binders for iron ore pelletization. In: Elsevier. Colloids and Surfaces A: Physicochem. 2003.

3 Sivrikaya O, Arol, A.I. Pelletization of magnetite ore with colemanite added organic binders. In: Powder Technology, 2011; (210): 23-28

4 Aragão RV, Pereira C, Rangel EP, Gouveia JF, Araújo DR, Fonseca MC, Costa RVP.Desenvolvimento de aglomerantes orgânicos na Samarco Mineração S/A. In: Seminário de Redução de Minério de Ferro. São Paulo: 2000; p. 147-148

5 Eisele TC, Kawatra SK. A Review of Binders in Iron Ore Pelletization. In: Mineral Processing \& Extractive Metall. Rev. Taylor \& Francis, 2003; p.1-90.

6 Haas LA. Effectiveness of organic binders for iron ore pelletization. University of Michigan, Michigan, 1989.

7 Honarkar H, Barikani M. Applications of biopolymers I: chitosan. In: Springer-Verlag, 2009. p. 1403-1420,

8 Šostar S, Schneider RA. study of fabric stiffness with guar gum in reactive printing. In: Dyes and Pigments, 1999; (41): 167-175.

* Contribuição técnica ao $44^{\circ}$ Seminário de Redução de Minério de Ferro e Matérias-primas, 15ํ Simpósio Brasileiro de Minério de Ferro e $2^{\circ}$ Simpósio Brasileiro de Aglomeração de Minério de Ferro, 15 a 18 de setembro de 2014, Belo Horizonte, MG, Brasil. 